\title{
Pulmonary tuberculosis among young children with severe pneumonia at Al Sabah Children's Hospital, Juba, South Sudan
}

\author{
Amanda Billy Berto Madison', Grace \\ Ndeezi $^{2}$, Hassen Chollong ${ }^{3}$, Justin \\ Bruno Tongun ${ }^{1}$, James K. Tumwine ${ }^{2}$, \\ Thereza Piloya ${ }^{2}$ \\ 1. Department of Paediatrics and \\ Child Health, School of Medicine, \\ University of Juba, Juba, South \\ Sudan \\ 2. Department of Paediatrics and Child \\ Health, College of Health Sciences, \\ School of Medicine, Makerere \\ University, Kampala, Uganda \\ 3. Al Sabah Children's Hospital, Juba, \\ South Sudan.
}

Correspondence:

Amanda Billy Berto Madison madisonamanda2009@gmail.com

Submitted: May 2021

Accepted: July 2021

Published: August 2021

\begin{abstract}
Background: Tuberculosis is a major public health problem worldwide. It can present in an acute form especially in endemic settings, which might lead to missed and delayed diagnosis, prolonged hospital stays, and increased mortality in children. South Sudan has a high prevalence of tuberculosis in the adult population. However, there is no published data on paediatric tuberculosis. We aimed to determine the prevalence and factors associated with pulmonary tuberculosis in children admitted with pneumonia in Al Sabah Children's Hospital, South Sudan.
\end{abstract}

Method: This was a cross sectional study of 404 children aged 2 to 59 months admitted with severe pneumonia from June-October 2018 at Al Sabah Children's Hospital. We excluded children on anti-TB treatment. Data were collected using a pretested questionnaire which captured socio-demographic characteristics, clinical history, physical examination, and laboratory investigations. Sputum examination for Mycobacterium tuberculosis was performed for all participants using X-pert MTB/RIF. Additional investigations included Chest X-ray and blood count.

Results: We recruited 404 children, out of which 78 (19.3\%) had pulmonary tuberculosis. Of these, 13 (16.7\%) were bacteriologically confirmed while 65 $(83.3 \%)$ were clinically diagnosed. The factors significantly associated with pulmonary tuberculosis were age above two years [AOR 2.32 (95\% CI 1.045.17)] p value 0.039, positive HIV Status [AOR 24.2 (95\% CI 2.88-202.62)] $p$ value 0.003 , severe acute malnutrition [AOR 15.67 (95\% CI 6.68-36.73)] $\mathrm{p}$ value $<0.001$, lack of BCG immunization [AOR 3.09 (95\% CI 1.06-9.03)] p value 0.038 , and contact with a known tuberculosis patient [AOR $55.14(95 \%$ CI 10.12-300.6)] p value $<0.001$.

Conclusion: There is a high burden of pulmonary tuberculosis in children presenting with severe pneumonia. There is a need for screening for pulmonary tuberculosis in children with any of the associated factors to improve early diagnosis and treatment.

Key words: Severe paediatric pneumonia, pulmonary tuberculosis (PTB), South Sudan

\section{Introduction}

Tuberculosis (TB) is a major public health problem globally. It ranks fourth among the top infectious disease killers, after acute respiratory infections, diarrhoeas, and human immunodeficiency virus (HIV) infection/acquired immunodeficiency syndrome (AIDS).$^{[1]}$ According to World Health Organization (WHO), approximately 10 million people developed TB in 2017, of which 1.0 million $(10 \%)$ were children. ${ }^{[2]}$ 
Pneumonia is a major cause of morbidity and mortality in infants and young children. It is the single largest infectious cause of death in children worldwide and it accounts for $16 \%$ of all deaths of children under five years old. ${ }^{[3-6]}$ Some deaths from pneumonia may follow delayed diagnosis of TB since TB presentation can mimic severe pneumonia from other causes especially in infants and young children. Most deaths due to pneumonia (70\%) occur in Southeast Asia and sub-Saharan Africa where TB is endemic. ${ }^{[6]}$

In 2014, sub-Saharan African countries reported a TB prevalence of 281 per 100,000 of the population which is more than double the global average of 133 per $100,000{ }^{[7]}$ A study in Uganda reported a reduction in childhood TB incidence in Kampala between 2009 and 2010. However, childhood TB cases were much higher at $7.5 \%$ during the same period compared to the national average of $2.5 \%$ in the world TB reports of 2010 and 2011. ${ }^{[8]}$

South Sudan is among the top 15 countries with the highest pneumonia related mortality

accounting for $20 \%$ of deaths due to pneumonia. ${ }^{[9,10]} \mathrm{TB}$ is endemic in South Sudan with a prevalence twice the global prevalence of 133 per $100,000{ }^{[11]}$ According to the National TB Programme (NTP), TB notification has increased from 2,955 cases in 2008 to 8,856 in 2014. In addition, the incidence of notified smearpositive pulmonary TB (PTB) cases was 37 per 100,000 population. ${ }^{[11]}$ Two thirds of TB cases were identified in four of the ten States of South Sudan with Central Equatorial State carrying the highest burden (38\%). ${ }^{[1]}$ However, there was no information regarding paediatric TB.

South Sudan is one of the countries with poor health indicators because of the ongoing conflict, violence, instability and underdevelopment. People are internally displaced and it has been estimated that one in four people in South Sudan, mainly children and women, had been forced to flee their homes. This subjected them to vulnerability to diseases without reliable access to medical care. The rationale for this study was to generate data to fill the knowledge gap on the burden of PTB in the paediatric population in South Sudan. The generated data will be used to develop strategies and guide healthcare workers to support these children effectively.

\section{Method}

This was a cross sectional study from June to October 2018 at Al Sabah Children's Hospital.

Al Sabah Children's Hospital, the only one in the country, is located in Central Equatoria State, in Juba. It remains the only facility specializing in a number of paediatric services, including an expanded programme of immunization
(EPI), treatment of severe acute malnutrition with medical complications, outpatient and inpatients medical services, and comprehensive HIV services. The hospital receives patients from all over the country.

Participants were children aged 2 to 59 months who fulfilled the WHO case definition for severe pneumonia. The children, whose caretakers gave informed consent, were consecutively enrolled. Very sick patients were stabilized before enrolment. Those on anti TB treatment were excluded. A pre-tested questionnaire was administered by the principal investigator (PI) and a trained research assistant. Demographic characteristics and risk factors for PTB were obtained from the caretakers.

A thorough examination was carried by the PI and/or a medical doctor who was the study coordinator. Weight and length were taken using calibrated standard Salter scale and stadiometer accurate to $100 \mathrm{~g}$ and $0.5 \mathrm{~cm}$ respectively. Nutritional status was assessed using the WHO gender specific weight/ height $Z$ score.

\section{Laboratory investigations}

Complete blood count was done using Beckman Coulter Act Diff 5 CP (Cap Pierce) Haematological Analyzer at Oromo Clinical and Diagnostic Laboratory. HIV serology was carried out on participants above 18 months old and on the mothers for the participants who were below 18 months. Participants who had other relatives as their primary caretakers were tested for HIV using the rapid diagnostic kits regardless of their age. Sputum induction

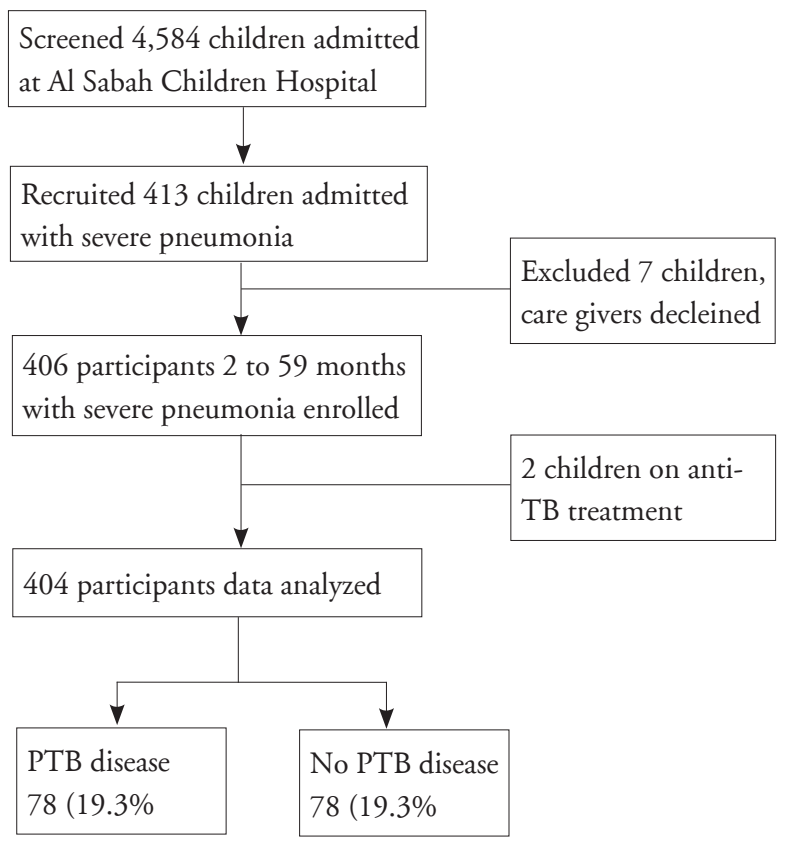

Figure 1: Study participant recruitment flow chart 
Table 1. Demographic characteristics of children aged 2 to 59 months presenting with severe pneumonia ( $N=404)$

\begin{tabular}{|c|c|}
\hline Factors & n (\%) \\
\hline \multicolumn{2}{|l|}{ Age } \\
\hline 2 - 24 months & $301(74.5)$ \\
\hline 25 - 59 months & $103(25.5)$ \\
\hline \multicolumn{2}{|l|}{ Sex } \\
\hline Female & $221(54.7)$ \\
\hline Male & $183(45.3)$ \\
\hline \multicolumn{2}{|c|}{ *Payam of residence } \\
\hline Juba & $49(12.1)$ \\
\hline Kator & $76(18.8)$ \\
\hline Munuki & 149(36.9) \\
\hline *Others & $130(32.2)$ \\
\hline \multicolumn{2}{|l|}{ HIV status } \\
\hline Positive & $13(3.2)$ \\
\hline Negative & $391(96.8)$ \\
\hline \multicolumn{2}{|c|}{ Previous history of pneumonia } \\
\hline Yes & $114(28.2)$ \\
\hline No & $290(71.8)$ \\
\hline \multicolumn{2}{|c|}{ BCG immunization status (Vaccine) } \\
\hline Yes & $378(93.6)$ \\
\hline No & $26(6.4)$ \\
\hline \multicolumn{2}{|c|}{ BCG Immunization Status (Scar) } \\
\hline Yes & $352(93.1)$ \\
\hline No & $26(6.9)$ \\
\hline \multicolumn{2}{|c|}{ Contact with known TB patient } \\
\hline Yes & $71(17.6)$ \\
\hline No & $333(82.4)$ \\
\hline
\end{tabular}

\begin{tabular}{|c|c|}
\hline \multicolumn{2}{|c|}{ Contact with chronic cough person } \\
\hline Yes & $97(24.0)$ \\
\hline No & $307(76.0)$ \\
\hline \multicolumn{2}{|l|}{ Fever } \\
\hline Yes & $394(97.5)$ \\
\hline No & $10(2.5)$ \\
\hline \multicolumn{2}{|l|}{ Lymph nodes enlargement } \\
\hline Yes & $112(27.7)$ \\
\hline No & $292(72.2)$ \\
\hline \multicolumn{2}{|c|}{ Nutritional status W/H Z score (SD) } \\
\hline Normal/Mild (>-1 to -2 ) & 256(63.3) \\
\hline *MAM (<-2 to -3$)$ & 49(12.1) \\
\hline *SAM (<-3) & $99(24.5)$ \\
\hline \multicolumn{2}{|l|}{$\mathrm{Hb}(\mathrm{g} / \mathrm{dl})$} \\
\hline Normal $\geq 11$ & $59(14.6)$ \\
\hline Mild anaemia 10-10.9 & $91(22.5)$ \\
\hline Moderate anaemia 7 to 9.9 & 205(50.7) \\
\hline Severe anaemia $<7$ & $49(12.1)$ \\
\hline \multicolumn{2}{|l|}{ Gene X-pert } \\
\hline Positive & $13(3.2)$ \\
\hline Negative & 391(96.7) \\
\hline \multicolumn{2}{|l|}{ Chest X-ray } \\
\hline Normal & $301(74.5)$ \\
\hline Abnormal & $83(20.5)$ \\
\hline Poor quality & $20(5)$ \\
\hline
\end{tabular}

*Payam is the fourth administrative level in government structure.

*Others; Northern Bari, Rajaf, Kondokoro.

*MAM = moderate acute malnutrition, SAM = severe acute malnutrition

was undertaken in all cases after explaining the procedure and its safety to the caretaker and after obtaining informed consent. The samples were transported to Juba Teaching Hospital laboratory where they were processed on the X-pert MTB/RIF Cepheid 4 module machine platform.

\section{Radiological investigations}

All participants had antero-posterior chest radiographs taken in two different centres The X-rays were read for features of TB by two independent radiologists and a radiology technician.

The main outcome measure in this study was the diagnosis of pulmonary TB.

\section{Operational definitions of a pulmonary TB case}

Bacteriologically confirmed PTB are those with positive gene X-pert MTB/RIF

Clinically diagnosed PTB:

- In HIV uninfected patient: At least two of the following: physical signs and symptoms suggestive of PTB, CXR consistent with PTB, history of exposure to TB patient.

- In HIV infected patients: At least one of the followings: contact with smear or X-pert positive TB patient, physical signs and symptoms or CXR suggestive of PTB. 
Table 2. Bivariate analysis of factors associated with PTB in children presenting with severe pneumonia

\begin{tabular}{|c|c|c|c|c|c|}
\hline Factor & Total $\mathbf{n}(\%)$ & No PTB & PTB (\%) & $\begin{array}{l}\text { Unadjusted Odds } \\
\text { Ratio (95\% CI) }\end{array}$ & p-value \\
\hline \multicolumn{6}{|l|}{ Child Factors } \\
\hline \multicolumn{6}{|l|}{ Age in months } \\
\hline $2-24$ & $301(74.5)$ & $252(83.7)$ & $49(16.3)$ & & \\
\hline $25-59$ & $103(25.5)$ & $74(71.8)$ & $29(28.2)$ & 2.02(1.19-3.41) & 0.009 \\
\hline \multicolumn{6}{|l|}{ Sex } \\
\hline Female & $221(54.7)$ & $179(81)$ & $42(19)$ & & \\
\hline Male & $183(45.3)$ & $147(80.3)$ & $36(19.7)$ & $1.04(0.64-1.71)$ & 0.866 \\
\hline \multicolumn{6}{|l|}{ HIV Status } \\
\hline Negative & $391(96.8)$ & $323(82.6)$ & $68(17.4)$ & & \\
\hline Positive & $13(3.2)$ & $3(23.1)$ & 10(76.9) & $15.83(4.24-59.06)$ & $<0.001$ \\
\hline \multicolumn{6}{|l|}{ WBC $\left(\times 10^{\wedge} 3 / \mu \mathrm{L}\right)$} \\
\hline$<12$ & $127(31.4)$ & $107(84.3)$ & $20(15.75)$ & & \\
\hline$>12$ & $277(68.6)$ & $219(79.1)$ & $58(20.9)$ & $1.42(0.91-2.48)$ & 0.221 \\
\hline \multicolumn{6}{|l|}{ Previous Pneumonia } \\
\hline No & 290(71.8) & $235(81)$ & $55(19)$ & & \\
\hline Yes & $114(28.2)$ & $91(79.8)$ & $23(20.2)$ & $1.08(0.63-1.86)$ & 0.782 \\
\hline \multicolumn{6}{|c|}{ BCG Immunization Status (Scar) } \\
\hline Yes & $352(93.1)$ & $290(82.4)$ & $62(17.6)$ & & \\
\hline No & $26(6.9)$ & $16(61.5)$ & $10(38.5)$ & $2.92(1.27-6.75)$ & 0.012 \\
\hline \multicolumn{6}{|c|}{ Nutritional Status W/H Z score (SD) } \\
\hline Normal/Mild >-1to -2 & $256(63.3)$ & 234(91.4) & $22(8.6)$ & & \\
\hline MAM <-2 to -3 & 49(12.1) & $45(91.8)$ & $4(8.2)$ & $0.95(0.31-2.87)$ & 0.921 \\
\hline SAM $<-3$ & $99(24.5)$ & $47(47.5)$ & $52(52.5)$ & $11.77(6.53-21.20)$ & $<0.001$ \\
\hline \multicolumn{6}{|l|}{ Maternal Factors } \\
\hline \multicolumn{6}{|l|}{ Educational Level } \\
\hline$\geq$ Secondary & $78(19.3)$ & 70(89.7) & $8(10.3)$ & & \\
\hline$<$ Secondary & $326(80.7)$ & $256(78.5)$ & $70(21.5)$ & $2.39(1.10-5.21)$ & 0.028 \\
\hline \multicolumn{6}{|l|}{ Employment Status } \\
\hline Housewife & $270(66.8)$ & $221(81.9)$ & $49(18.2)$ & & \\
\hline Self Employed & $108(26.7)$ & $85(78.7)$ & $23(21.3)$ & $1.22(0.7-2.13)$ & 0.482 \\
\hline Formal Employment & $26(6.4)$ & 20(76.9) & $6(23.1)$ & $1.35(0.52-3.55)$ & 0.538 \\
\hline \multicolumn{6}{|c|}{$\begin{array}{l}\text { Household Cigarette/ Shisha } \\
\text { Smoking }\end{array}$} \\
\hline No & $206(51)$ & $170(82.5)$ & $36(17.5)$ & & \\
\hline Yes & 198(49) & $156(78.8)$ & $42(21.2)$ & $1.27(0.77-2.09)$ & 0.342 \\
\hline
\end{tabular}




\begin{tabular}{|c|c|c|c|c|c|}
\hline \multicolumn{6}{|c|}{ Environmental Factors } \\
\hline \multicolumn{6}{|c|}{ Contact with known TB patient } \\
\hline No & $333(82.4)$ & $298(89.5)$ & $35(10.5)$ & & \\
\hline Yes & $71(17.6)$ & $28(39.4)$ & $43(60.6)$ & $13.08(7.24-23.61)$ & $<0.001$ \\
\hline \multicolumn{6}{|c|}{ Contact with chronic cough person } \\
\hline No & $307(76.0)$ & $269(87.6)$ & $38(12.4)$ & & \\
\hline Yes & $97(24.0)$ & $57(58.8)$ & $40(41.2)$ & $4.97(2.93-8.42)$ & $<0.001$ \\
\hline
\end{tabular}

\section{Statistical analysis}

The Kish-Leslie formula was used to calculate the sample size. The minimum sample size derived for objective one (burden of PTB in children with severe pneumonia) was 403. This sample size was sufficient to observe statistical significance at a 95\% level of confidence for both study objectives. A minimum sample size of 116 with at least 58 TB cases was sufficient to explore associated factors.

The data were entered into a computer using Microsoft Access 2016 and was analysed using STATA version 15. For continuous variables, means (SD) and medians (IQR) were used. For categorical variables proportions and percentages were used. Multivariate analysis using logistic regression was carried out on variables with p-value of less than 0.05 for bivariate analysis, to adjust for confounding and collinearity. Variables with a p-value of less 0.05 were considered significant at multivariate.

\section{Results}

\section{Participant demographic characteristics}

We screened 4,584 children aged 2 to 59 months, 406 children with severe pneumonia were enrolled and data for 404 were analysed. (Figure 1). The median age was 14 months; $54.7 \%$ of the participants were females; $36.9 \%$ were from Munuki Payam; 3.2\% were HIV positive. Among these, only one was internally displaced. The majority of the participants (97.5\%) had fever. Most had no lymphadenopathy; $24.5 \%$ were severely malnourished. Abnormal chest X-ray findings (airway compression, soft tissue density suggestive of lymph nodes, air space opacification, nodular picture, pleural effusion, cavities) were reported in $20.5 \%$. Socio-demographic characteristics are summarized in Table 1.

\section{Prevalence of PTB in children presenting with severe pneumonia}

The prevalence of PTB among children aged 2 to 59 months presenting with severe pneumonia was $19.3 \%$. Sputum induction for gene X-pert test was performed to all participants, however, only $16.7 \%$ who were diagnosed with PTB had positive results. While 83.3\% of the participants were clinically diagnosed through a combination of symptoms and signs consistent with $\mathrm{TB}$, contact with known TB patient, and chest X-ray suggestive of TB.

\section{Factors associated with pulmonary TB in children presenting with severe pneumonia}

In children with severe pneumonia, significant clinical association with pulmonary $\mathrm{TB}$ at bivariate analysis were found in the following subgroups: age above 24 months, positive HIV status, absence of scar of BCG vaccination, severe acute malnutrition, maternal educational level below secondary, and contact with both chronic cough and known TB patient (Table 2). Using multivariate analysis, the factors that remained independently associated with PTB were: age above 24 months, positive HIV status, absence of scar of BCG vaccination, and contact with known TB patient (Table 3).

\section{Discussion}

The prevalence of PTB in children in this study was one child in every five cases of severe pneumonia. This showed that pneumonia is a major public health problem in children under five years of age in South Sudan. It is one of the major causes of hospital admissions for children and accounts for $20 \%$ of the mortality in the under five-yearolds. ${ }^{[9]}$ Some of the mortality due to pneumonia could be attributed to PTB since the presentation of these two diseases are similar. This might result in delay in diagnosis, prolonged hospital stays and increase mortality.

The high prevalence of PTB might reflect the ongoing transmission in the community since children acquire the infection from the adult population. The prevalence found in this study is similar to $18.9 \%$ recorded in Uganda. ${ }^{[6]}$ These studies were done in children with severe pneumonia, in an urban setting and in government referral public hospitals. The children who visited these health facilities were mostly of low socio-economic status who were unable to afford private care. However, our finding is lower than the $23 \%$ prevalence reported in a study in 
Table 3. Multivariate Analysis of Factors associated with PTB in children presenting with severe pneumonia

\begin{tabular}{|c|c|c|c|c|}
\hline \multirow[b]{2}{*}{ Factors } & \multicolumn{2}{|l|}{ Bivariate } & \multicolumn{2}{|l|}{ Multivariate Model } \\
\hline & $\begin{array}{l}\text { Unadjusted Odds } \\
\text { Ratio }(95 \% \mathrm{Cl})\end{array}$ & $p$-value & $\begin{array}{l}\text { Adjusted Odds Ratio } \\
(95 \% \mathrm{Cl})\end{array}$ & p-value \\
\hline \multicolumn{5}{|l|}{ Child Factors } \\
\hline \multicolumn{5}{|l|}{ Age in months } \\
\hline $2-24$ & 1 & & & \\
\hline $25-59$ & $2.02(1.19-3.41)$ & 0.009 & $2.32(1.04-5.17)$ & 0.039 \\
\hline \multicolumn{5}{|l|}{ HIV Status } \\
\hline Negative & 1 & & & \\
\hline Positive & $15.83(4.24-59.06)$ & 0.000 & $24.2(2.88-202.62)$ & 0.003 \\
\hline \multicolumn{5}{|c|}{ Nutrition Status W/H Z score (SD) } \\
\hline Normal/Mild $>-1$ to -2 & 1 & & & \\
\hline$M A M<-2$ to -3 & $0.95(0.31-2.87)$ & 0.921 & $0.822(0.23-2.98)$ & \\
\hline SAM $<-3$ & $11.77(6.53-21.20)$ & 0.000 & $15.67(6.68-36.73)$ & $<0.001$ \\
\hline \multicolumn{5}{|l|}{ Maternal Factors } \\
\hline \multicolumn{5}{|l|}{ Educational Level } \\
\hline$\geq$ Secondary & 1 & & & \\
\hline$<$ Secondary & $2.39(1.1-5.21)$ & 0.028 & $1.35(0.47-3.92)$ & 0.575 \\
\hline \multicolumn{5}{|l|}{ Health System Factors } \\
\hline \multicolumn{5}{|c|}{ BCG Immunization Status (Scar) } \\
\hline Yes & 1 & & & \\
\hline No & $2.92(1.27-6.75)$ & 0.012 & $3.09(1.06-9.03)$ & 0.038 \\
\hline \multicolumn{5}{|l|}{ Environmental Factors } \\
\hline \multicolumn{5}{|c|}{ Contact with known TB Patient } \\
\hline No & 1 & & & \\
\hline Yes & $13.08(7.24-23.61)$ & 0.000 & $55.14(10.12-300.6)$ & $<0.001$ \\
\hline \multicolumn{5}{|c|}{ Contact with Chronic Cough Person } \\
\hline No & 1 & & & \\
\hline Yes & $4.97(2.93-8.42)$ & 0.000 & $0.35(0.68-1.84)$ & 0.216 \\
\hline
\end{tabular}

Bangladesh. The differences could be attributed to the fact that the study in Bangladesh enrolled malnourished children who had severe pneumonia. ${ }^{[4]}$ Malnutrition is a known cause of immune suppression and it increases the risk for both progression of TB infection and reactivation of latent TB.

\section{Factors associated with PTB in children presenting with severe pneumonia}

In this study, age above two years was associated with PTB. Age of the child affects its risk of TB exposure as older children interact more with adults. They may be exposed to infectious cases of TB at home or in the community. On the other hand, younger children, especially infants, interact with fewer adults in their family units suggesting that contact could be other community members apart from their mothers. Our findings are similar to a study done by Robin et al in South Africa which showed 10\% of TB cases notified to the national tuberculosis control program were of children aged less than fifteen years. Of these, $66 \%$ were less than five years. ${ }^{[12]}$ Other studies done in Uganda and Congo showed contrary results where they found that TB is more likely to occur in children under two years of age. ${ }^{[6,13]}$ 
HIV positive children were 24 times more likely to develop PTB compared to their counterparts who were HIV negative. HIV destroys CD4+ cell and T cell mediated immunity which is critical in fighting against TB bacilli. In the absence of $\mathrm{CD} 4+$ cells, the body remains vulnerable to a wide range of infections including TB. Our finding is consistent with other studies which found that HIV is a risk factor for paediatric TB.[14,15] In addition, HIV prevalence among children with TB was found to be as high as $60 \%$ in countries with high HIV prevalence.[16] Further, another study in Uganda found a prevalence of $49 \%$ of TB/HIV in an area of high burden of paediatric HIV. However, another study in Uganda found no association between HIV and TB. ${ }^{[6,17]}$

Children who were severely malnourished were 16 times more likely to suffer from PTB than those who had normal nutritional status. Severe acute malnutrition is a known cause of immune suppression affecting both innate and adaptive immune responses. Malnutrition also causes thymic atrophy which results in depletion of CD4+, CD8+ and thymocytes and increases the risk for infections. These findings are in keeping with a study done by Chisti et al and a review article by Padmanesan et al. ${ }^{[18 \text {, }}$ ${ }^{19]}$ A study by Nantongo et al found a 35\% prevalence in children who had both moderate and severe acute malnutrition. ${ }^{[6]}$

Children who had not received BCG immunization were three times more likely to develop PTB compared to those who had received the vaccine. Recent studies found $B C G$ vaccination to be protective against $T B$ infection. ${ }^{[20,21]}$ Two different systematic reviews also found BCG vaccination to be protective against TB. ${ }^{[21,22]}$ Furthermore, there is evidence that supports vaccinating children soon after birth to prevent infection and disease especially in countries with a high TB burden. ${ }^{[21,22]}$ However, the findings of our study is contrary to studies done in Uganda and India which found no association between BCG immunization and TB. ${ }^{[6,14]}$

Contact with patients who had tuberculosis was found to be associated with PTB. This is important finding because paediatric $\mathrm{TB}$ reflects the ongoing transmission in the community and children represent $10 \%$ of overall TB prevalence. On the other hand, isoniazid prophylaxis was found to be effective in reducing the risk of developing tuberculosis among children who had positive $\mathrm{TB}$ contacts by $59 \% \cdot{ }^{[23]}$ In this study, none of the participants who had positive contacts with known TB patients were given INH prophylaxis. Findings of this study are similar to that reported in Uganda and India. ${ }^{[4,6]}$ Other studies have also described the association between contact with TB cases and development of TB disease in children. ${ }^{[14,19]}$

Displacement, both internally and externally, is known to increase the risk of airborne infections such as TB. This results from increase in population density, disruption and poor access to health facilities. ${ }^{[28]}$ In South Sudan, approximately $40 \%$ of the pre-war population are displaced either within the country or as refugees to the neighbouring countries. ${ }^{[29]}$ In this study, there was no association between PTB and displacement, only one of the participants was internally displaced. This could be attributed to the fact that most of the displaced population is still in the refugee camps and medical services are provided within the camps.

Several studies have measured the socio-economic status using parental educational level, income and occupation. $[24,25]$ In this study, low maternal education was the measure of low socio-economic status, and it suggested an association with PTB in children presenting with severe pneumonia but did not reach conventional significance. Low socio-economic status is associated with overcrowding, poor living conditions, poor knowledge and health seeking behaviour which in turn are associated with increased risk of diseases including TB. ${ }^{[26,27]}$

\section{Conclusion}

We found a high prevalence of PTB among children admitted with severe pneumonia at Al Sabah Children's Hospital in Juba, South Sudan. Children with severe pneumonia were more likely to have PTB if they were above two years of age, HIV positive, have severe acute malnutrition, have not received BCG immunization or had contact with known TB patients.

\section{Ethics approval and consent to participate}

Approval to conduct this study was obtained from the Department of Paediatrics and Child Health, Makerere University College of Health Sciences. Ethical approval was obtained from Makerere University College of Health Sciences School of Medicine Research Ethics Committee (SOMREC). Approval was also obtained from the Ministry of Health in the Republic of South Sudan and permission was sought from Al Sabah Children's Hospital in Juba, South Sudan.

Written informed consent was obtained from parents/ guardians of eligible participants. Consent forms for caregivers were translated into Arabic. For purposes of confidentiality only study specific serial numbers were used instead of names of the participants. The data were coded and accessible only to the research team.

\section{Consent for publication: Not applicable}

\section{Competing interests: None}

Funding: SURVIVAL-PLUSS project under NORHED with funding through NORAD- Norway and Gordon Memorial College Trust Fund

Acknowledgements: We thank all the care-takers, 
patients and staff of Al Sabah Children's Hospital.

\section{References}

1. World Health Organization, Geneva. Global Tuberculosis Report 2015. https://apps.who.int/ iris/handle/10665/191102

2. World Health Organization, Geneva. Global Tuberculosis Report 2018. https://apps.who.int/ iris/handle/10665/274453

3. Abuka T. Prevalence of pneumonia and factors associated among children 2-59 months old in Wondo Genet district, Sidama zone, SNNPR, Ethiopia. Current Pediatric Research. 2017.

4. Chisti MJ, Graham SM, Duke T, Ahmed T, Ashraf H, Faruque ASG, et al. A prospective study of the prevalence of tuberculosis and bacteraemia in Bangladeshi children with severe malnutrition and pneumonia including an evaluation of Xpert MTB/RIF assay. PloS one. 2014;9(4):e93776.

5. Oliwa JN, Karumbi JM, Marais BJ, Madhi SA, Graham SM. Tuberculosis as a cause or comorbidity of childhood pneumonia in tuberculosis-endemic areas: a systematic review. The Lancet Respiratory Medicine 2015;3(3):235-43.

6. Nantongo JM, Wobudeya E, Mupere E, Joloba M, Ssengooba W, Kisembo HN, et al. High incidence of pulmonary tuberculosis in children admitted with severe pneumonia in Uganda. BMC pediatrics. 2013;13(1):16.

7. World Health Organization. Global tuberculosis report 2015. WHO/HTM/TB/2015.22. Geneva, WHO Press; 2015.

8. Wobudeya E, Lukoye D, Lubega IR, Mugabe F, Sekadde M, Musoke P. Epidemiology of tuberculosis in children in Kampala district, Uganda, 2009-2010; a retrospective cross-sectional study. BMC public health. 2015;15(1):967.

9. Malaria Consortium. http://www. malariaconsortium.org/projects/pneumoniadiagnostics/15/south-sudan

10. Abd-Elfarag GOE, Langoya CO. Household air pollution and childhood pneumonia in South Sudan: will clean cooking stoves reduce the incidence and mortality? South Sudan Medical Journal. 2016;9(2):36-9.

11. Joseph Lasu JL, Salah-Eddine Ottmani, Macharia $S$, et al. Guideline for Tuberculosis \& TB/HIV prevention, care and control Third ed 2016. p2 $\& 3$.
12. Wood R, Johnstone-Robertson S, Uys P, Hargrove J, Middelkoop K, Lawn SD, et al. Tuberculosis transmission to young children in a South African community: modeling household and community infection risks. Clinical infectious diseases. 2010;51(4):401-8.

13. Nika ER, Mabiala Babela JR, Missambou Mandilou SV, Moyen G. Study of 9 Cases of Tuberculosis Pneumonia in Children at Chu of Brazzaville, Congo. Global pediatric health. 2016;3:2333794X16651512.

14. Jain SK, Ordonez A, Kinikar A, Gupte N, Thakar M, Mave V, et al. Pediatric tuberculosis in young children in India: a prospective study. BioMed research international. 2013;2013.

15. Dodd P, Prendergast A, Beecroft C, Kampmann $\mathrm{B}$, Seddon J. The impact of HIV and antiretroviral therapy on TB risk in children: a systematic review and meta-analysis. Thorax. 2017;72(6):559-75.

16. Venturini E, Turkova A, Chiappini E, Galli L, de Martino M, Thorne C. Tuberculosis and HIV co-infection in children. BMC infectious diseases. 2014;14(1):S5.

17. Iriso R. The clinical, radiological and laboratory features of tuberculosis in children at Mulago Hospital. 2002.

18. Narasimhan P, Wood J, MacIntyre CR, Mathai D. Risk factors for tuberculosis. Pulmonary medicine. 2013;2013.

19. Chisti MJ, Ahmed T, Shahid AS, Shahunja K, Bardhan PK, Faruque ASG, et al. Sociodemographic, Epidemiological, and Clinical Risk Factors for Childhood Pulmonary Tuberculosis in Severely Malnourished Children Presenting With Pneumonia: Observation in an Urban Hospital in Bangladesh. Global pediatric health. 2015;2:2333794X15594183.

20. Dockrell HM, Smith SG. What have we learnt about BCG vaccination in the last 20 years? Frontiers in immunology. 2017;8:1134.

21. Roy A, Eisenhut M, Harris R, Rodrigues L, Sridhar $S$, Habermann S, et al. Effect of BCG vaccination against Mycobacterium tuberculosis infection in children: systematic review and meta-analysis. Bmj. 2014;349:g4643.

22. Mangtani P, Abubakar I, Ariti C, Beynon R, Pimpin L, Fine PE, et al. Protection by BCG vaccine against tuberculosis: a systematic review of randomized controlled trials. Clinical infectious diseases. 2013;58(4):470-80. 
23. Ayieko J, Abuogi L, Simchowitz B, Bukusi EA, Smith AH, Reingold A. Efficacy of isoniazid prophylactic therapy in prevention of tuberculosis in children: a meta-analysis. BMC infectious diseases. 2014;14(1):91.

24. Chen Q, Kong Y, Gao W, Mo L. Effects of Socioeconomic Status, Parent-Child Relationship, and Learning Motivation on Reading Ability. Frontiers in psychology. 2018;9.

25. Sirin SR. Socioeconomic status and academic achievement: A meta-analytic review of research. Review of educational research. 2005;75(3):41753.

26. Gupta D, Das K, Balamughesh T, Aggarwal N, Jindal SK. Role of socio-economic factors in tuberculosis prevalence. Indian Journal of Tuberculosis. 2004;51(1):27-32.
27. Jiamsakul A, Lee M, Nguyen K, Merati T, Cuong $\mathrm{D}$, Ditangco R, et al. Socio-economic status and risk of tuberculosis: a case-control study of HIV-infected patients in Asia. The International Journal of Tuberculosis and Lung Disease. 2018;22(2):179-86.

28. Hosten E, Mehta M, Andre E, Rumman KA, Van der Linden D. Tuberculosis contact-tracing among Syrian refugee populations: lessons from Jordan. Conflict and health. 2018;12(1):25.

29. Campbell GBfJ. https://www.cfr.org/blog/despitepeace-deal-too-dangerous-south-sudanese-idpsreturn-home 2018. 